\title{
COMMON FIXED POINT THEOREM IN FUZZY METRIC SPACE USING CONTROL FUNCTION
}

\author{
Amit Kumar and Ramesh Kumar Vats
}

\begin{abstract}
We give a fixed point theorem for complete fuzzy metric space which generalizes fuzzy Banach contraction theorems established by V. Gregori and A. Spena [Fuzzy Sets and Systems 125 (2002), 245-252] using notion of altering distance, initiated by Khan et al. [Bull. Austral. Math. Soc. 30 (1984), 1-9] in metric spaces.
\end{abstract}

\section{Introduction}

Unless mentioned or defined otherwise, for all terminology and notation in this paper, the reader is referred to $[2,3,4,5,6,7,8,9,10,12,13,15]$.

Kramosil and Michalek [12] introduced fuzzy metric space, George and Veermani [8] modified the notion of fuzzy metric spaces with the help of continuous $t$-norms. George and Veeramani [8] imposed some stronger conditions on the fuzzy metric space in order to obtain a Hausdorff topology. The aim of this paper is to generalize the Banach fixed point theorem to (fuzzy) contractive mappings on complete fuzzy metric spaces in George and Veeramani sense using concept of alternating distance.

\section{Preliminaries}

In what follows, we collect some relevant definitions, results, examples for our further use.

Definition 2.1 (Schweizer and Sklar [15]). A binary operation $*:[0,1] \times$ $[0,1] \rightarrow[0,1]$ is a continuous $t$-norm, if $([0,1], *)$ is a topological monoid with unit 1 such that $a * b \leq c * d$ whenever $a \leq c$ and $b \leq d(a, b, c, d \in[0,1])$.

Definition 2.2. A continuous t-norm (in sense of Schweizer and Sklar [15]) is a binary operation $T$ on $[0,1]$ satisfying the following conditions:

- $T$ is commutative and associative;

- $T(a, 1)=a$ for all $a \in[0,1]$;

Received July 10, 2012

2010 Mathematics Subject Classification. Primary 47H10, 54E35; Secondary 54H25.

Key words and phrases. common fixed point, fuzzy contractive mapping, complete fuzzy metric space. 
- $T(a, b)=T(c, d)$ whenever $a=c$ and $b=d(a, b, c, d \in[0,1])$;

- The mapping $T:[0,1] \times[0,1] \rightarrow[0,1]$ is continuous.

Definition 2.3. A fuzzy metric space (in sense of Kramosil and Michalek [12]) is a triple $(X, M, *)$, where $X$ is a nonempty set, $*$ is a continuous $t$-norm and $M$ is a fuzzy set on $X^{2} \times[0, \infty)$ such that the following axioms hold:

- $M(x, y, 0)=0(x, y \in X)$;

- $M(x, y, t)=1$ for all $t>0$ if and only if $x=y$;

- $M(x, y, t)=M(y, x, t)(x, y \in X, t>0)$;

- $M(x, y, \cdot):[0, \infty) \rightarrow[0,1]$ is left continuous for all $x, y \in X$;

- $M(x, z, t+s) \geq M(x, y, t) * M(y, z, s)$ for all $x, y, z \in X$ and $s, t>0$.

We will refer to these spaces as KM-fuzzy metric spaces. To obtain a Hausdorff topology on the fuzzy metric space, the authors gave the following definitions in [8].

Definition 2.4. A fuzzy metric space (in sense of George and Veeramani [8]) is a triple $(X, M, *)$, where $X$ is a nonempty set, $*$ is a continuous $t$-norm and $M$ is a fuzzy set on $X^{2} \times(0, \infty)$ such that the following axioms hold:

- $M(x, y, t)>0(x, y \in X)$;

- $M(x, y, t)=1$ for all $t>0$ if and only if $x=y$;

- $M(x, y, t)=M(y, x, t)(x, y \in X, t>0)$;

- $M(x, y, \cdot):(0, \infty) \rightarrow(0,1]$ is continuous for all $x, y \in X$

- $M(x, z, t+s) \geq M(x, y, t) * M(y, z, s)$ for all $x, y, z \in X$ and $s, t>0$.

Notice that condition (last), is a fuzzy version of triangular inequality. The value $M(x, y, t)$ can be thought of as degree of nearness between $x$ and $y$ with respect to $t$ and from axiom (second), we can relate the value 0 and 1 of a fuzzy metric to the notions of $\infty$ and 0 of classical metric respectively.

Definition 2.5 (George and Veeramani [8]). Let $(X, M, *)$ be a fuzzy metric space. The open ball $B(x, r, t)$ for $t>0$ with centre $x \in X$ and radius $r$, $0<r<1$, is defined as $B(x, r, t)=\{y \in X: M(x, y, t)>1-r\}$. The family $\{B(x, r, t): x \in X ; 0<r<1, t>0\}$ is a neighborhood system for a Hausdorff topology on $X$, that we call induced by the fuzzy metric $M$.

Definition 2.6 (George and Veeramani [8]). In a metric space $(X, d)$ the 3tuple $\left(X, M_{d}, *\right)$ where $M_{d}(x, y, t)=\frac{t}{t+d(x, y)}$ and $a * b=a b$, is a fuzzy metric space. This $M_{d}$ is called the standard fuzzy metric induced by $d$. The topologies generated by the standard fuzzy metric and the corresponding metric are the same.

Definition 2.7 (George and Veeramani [8]). A sequence $\left\{x_{n}\right\}$ in a fuzzy metric space $(X, M, *)$ is a Cauchy sequence if and only if for each $\epsilon \in(0,1)$ and each $t>0$ there exists $n_{0} \in N$ such that $M\left(x_{n}, x_{m}, t\right)>1-\epsilon$ for all $n, m \geq n_{0}$. A fuzzy metric space in which every Cauchy sequence is convergent is called a complete fuzzy metric space. 
Definition 2.8 (G-Cauchy sequence $[5,6])$. A sequence $\left\{x_{n}\right\}$ in a fuzzy metric space $(X, M, *)$ is called a $G$-Cauchy if $\lim _{n \rightarrow \infty} M\left(x_{n}, x_{n+m}, t\right)=1$ for each $m \in N$ and $t>0$.

We call a fuzzy metric space $(X, M, *)$ is $G$-complete if every $G$-Cauchy sequence in $X$ is convergent. It follows immediately that a Cauchy sequence is a $G$-Cauchy sequence. The converse is not always true, this has been established by an example in [16].

The following concept of convergence was introduced in fuzzy metric spaces by Mihet [14].

Theorem 2.1 (George and Veeramani [8]). A sequence $\left\{x_{n}\right\}$ in a fuzzy metric space $(X, M, *)$ converges to $x$ if and only if $M\left(x_{n}, x, t\right) \rightarrow 1$ as $n \rightarrow \infty$.

V. Gregori and A. Sapena [10] established fixed point theorem for following type fuzzy contractive mappings.

Definition 2.9. Let $(X, M, *)$ be a fuzzy metric space. We will say the mapping $f: X \times X$ is fuzzy contractive if there exists $k \in(0,1)$ such that

$$
\frac{1}{M(f(x), f(y), t)}-1 \leq k\left(\frac{1}{M(x, y, t)}-1\right)
$$

for each $x, y \in X$ and $t>0$ ( $k$ is called the contractive constant of $f$ ).

Definition 2.10 (Altering distance function [11]). An altering distance function or control function is a function $\psi:[0, \infty) \rightarrow[0, \infty)$ such that the following axioms hold:

- $\psi$ is monotonic increasing and continuous;

- $\psi(t)=0$ if and only if $t=0$.

In 1984 using the altering distance function Khan et al. [11] proved the following result.

Theorem 2.2 ([11]). Let $(X, d)$ be a complete metric space, $\psi$ be an altering distance function and let $f: X \rightarrow X$ be a self mapping which satisfies the following inequality $\psi(d(f x, f y)) \leq c \psi(d(x, y))$ for all $x, y \in X$ and for some $0<c<1$. Then $f$ has a unique fixed point.

Definition 2.11. A function $\phi: \mathrm{R} \rightarrow R^{+}$is said to satisfy the condition $*$ if the following axioms hold:

- $\phi(t)=0$ if and only if $t=0$;

- $\phi(t)$ is increasing and $\phi(t) \rightarrow \infty$ as $t \rightarrow \infty$;

- $\phi$ is left continuous in $(0, \infty)$;

- $\phi$ is continuous at 0 .

In this connection B. S. Choudhury et al. [4] have been studied the fixed point results in Menger Space, for more details see in $[3,5,6]$. Recently C. T. Aage and B. S. Choudhury [1] has proved the following result. 
Theorem 2.3. Let $(X, M, T)$ be a fuzzy metric space in the sense of George and Veeramani and $\sup _{0=a<1} T(a, a)=1$ and the self mapping $f: X \rightarrow X$ satisfy $M(f x, f y, f(t)) \geq M\left(x, y, \phi\left(\frac{t}{c}\right)\right)$, where $0<c<1, x, y \in X$ and $t>0$ and $\phi$ satisfies $*$ condition. Suppose that for some $x_{0} \in X$ the sequence of $\left\{f^{n} x_{0}\right\}$ has a p-convergent subsequence. Then $f$ has a unique fixed point.

Recently, C. T. Aage and J. N. Salunke [2] proved existence and uniqueness of fixed point in fuzzy metric space using the following contraction

$$
\frac{1}{M(f x, f y, \phi(c t))}-1 \leq \psi\left(\frac{1}{M(x, y, t)}-1\right) .
$$

In this manuscript, we generalize contractive condition (2.1) using alternating distance and establish a fixed point theorem in $G$-complete fuzzy metric space in the sense of George and Veeramani.

\section{Main theorem}

Theorem 3.1. Let $(X, M, *)$ be a $G$-complete fuzzy metric space and $f, T$ : $X \rightarrow X$ be two self mappings satisfying

i) $T X \subseteq f X$;

ii) The functions $\psi, \alpha:[0,1) \rightarrow[0,1)$ are continuous, monotonically increasing with $\psi(0)=0=\alpha(0)$ and $t-\alpha(t)+\psi(t)>0$, also $(\alpha-$ $\psi)^{n}\left(a_{n}\right) \rightarrow 0$, whenever $a_{n} \rightarrow 0$ as $n \rightarrow \infty$;

iii) $M(f x, f y, \phi(t))>0 \forall t>0$ where the function $\phi$ satisfies Definition 2.11.

Also the contraction with above three conditions

$\frac{1}{M(T x, T y, \phi(c t))}-1 \leq \alpha\left(\frac{1}{M(f x, f y, \phi(t))}-1\right)-\psi\left(\frac{1}{M(f x, f y, \phi(t))}-1\right)$

holds for all $x, y \in X, t>0,0<c<1$.

If $f X$ is a G-complete subspace of $X$ and the mapping $(f, T)$ are weakly compatible, then $f$ and $T$ have a unique common fixed point.

Proof. Let $x_{0}$ be any point in $X$, we define two sequences $\left\{y_{n}\right\}$ and $\left\{x_{n}\right\}$ such that $y_{n}=T x_{n}=f x_{n+1}$, we claim that $\left\{y_{n}\right\}$ is a Cauchy sequence.

Assume that, if possible, for some $n$ the following is true

$$
\frac{1}{M\left(T x_{n-1}, T x_{n}, \phi(c t)\right)}-1 \leq \frac{1}{M\left(T x_{n}, T x_{n+1}, \phi(c t)\right)}-1 .
$$

On substituting $x=x_{n}, y=x_{n+1}$ in (3.2) we get,

$$
\begin{aligned}
& \frac{1}{M\left(T x_{n}, T x_{n+1}, \phi(c t)\right)}-1 \\
\leq & \alpha\left(\frac{1}{M\left(f x_{n}, f x_{n+1}, \phi(t)\right)}-1\right)-\psi\left(\frac{1}{M\left(f x_{n}, f x_{n+1}, \phi(t)\right)}-1\right) .
\end{aligned}
$$


Using the above assumption the contraction becomes

$$
\begin{aligned}
& \frac{1}{M\left(T x_{n-1}, T x_{n}, \phi(c t)\right)}-1 \\
\leq & \alpha\left(\frac{1}{M\left(T x_{n-1}, T x_{n}, \phi(t)\right)}-1\right)-\psi\left(\frac{1}{M\left(T x_{n-1}, T x_{n}, \phi(t)\right)}-1\right) .
\end{aligned}
$$

However, $t-\alpha(t)+\psi(t)>0$, which is contrary to our assumption. Thus, $\forall n$

$$
\frac{1}{M\left(T x_{n}, T x_{n+1}, \phi(c t)\right)}-1<\frac{1}{M\left(T x_{n-1}, T x_{n}, \phi(c t)\right)}-1 \text {. }
$$

Again, we assume that $\left\{y_{n}\right\} \neq\left\{y_{n+1}\right\} \forall n$. By virtue of the properties of $\phi$, we can find a $t>0$ such that $M\left(f x_{1}, f x_{2}, \phi(t)\right)>0$. Therefore using (3.2) we get

$$
\begin{aligned}
& \frac{1}{M\left(y_{0}, y_{1}, \phi(c t)\right)}-1 \\
= & \frac{1}{M\left(T x_{0}, T x_{1}, \phi(c t)\right)}-1 \\
\leq & \alpha\left(\frac{1}{M\left(f x_{1}, f x_{2}, \phi(t)\right)}-1\right)-\psi\left(\frac{1}{M\left(f x_{1}, f x_{2}, \phi(t)\right)}-1\right)
\end{aligned}
$$

on, using (3.3) the expression becomes

$$
\begin{aligned}
& \frac{1}{M\left(T x_{1}, T x_{2}, \phi(c t)\right)}-1 \\
\leq & \alpha\left(\frac{1}{M\left(f x_{1}, f x_{2}, \phi(t)\right)}-1\right)-\psi\left(\frac{1}{M\left(f x_{1}, f x_{2}, \phi(t)\right)}-1\right) .
\end{aligned}
$$

Again $M\left(f x_{1}, f x_{2}, \phi(t)\right)>0$ implies $M\left(f x_{1}, f x_{2}, \phi\left(\frac{t}{c}\right)\right)>0$ therefore by application of (3.2), we obtain

$$
\begin{aligned}
& \frac{1}{M\left(y_{0}, y_{1}, \phi(t)\right)}-1 \\
= & \frac{1}{M\left(T x_{0}, T x_{1}, \phi(t)\right)}-1 \\
\leq & \alpha\left(\frac{1}{M\left(f x_{1}, f x_{2}, \phi\left(\frac{t}{c}\right)\right)}-1\right)-\psi\left(\frac{1}{M\left(f x_{1}, f x_{2}, \phi\left(\frac{t}{c}\right)\right)}-1\right) .
\end{aligned}
$$

Again, by using (3.3) the equation turns out to be

$$
\begin{aligned}
& \frac{1}{M\left(T x_{1}, T x_{2}, \phi(t)\right)}-1 \\
\leq & \alpha\left(\frac{1}{M\left(f x_{1}, f x_{2}, \phi\left(\frac{t}{c}\right)\right)}-1\right)-\psi\left(\frac{1}{M\left(f x_{1}, f x_{2}, \phi\left(\frac{t}{c}\right)\right)}-1\right) .
\end{aligned}
$$


Repeating the above process $n$ times ones obtain

$$
\frac{1}{M\left(y_{n-1}, y_{n}, \phi(t)\right.}-1 \leq(\alpha-\psi)^{n}\left(\frac{1}{M\left(f x_{1}, f x_{2}, \phi\left(\frac{t}{c^{n}}\right)\right)}-1\right) .
$$

Since condition (iii) implies that $M\left(f x_{2}, f x_{3}, \phi(c t)\right)>0$ then following the above process we have

$$
\frac{1}{M\left(y_{n-1}, y_{n}, \phi(c t)\right)}-1 \leq(\alpha-\psi)^{n}\left(\frac{1}{M\left(f x_{2}, f x_{3}, \phi\left(\frac{c t}{c^{n}}\right)\right)}-1\right) .
$$

Continuing the above process $r$ times, we have for $n>r$

$$
\begin{aligned}
& \frac{1}{M\left(y_{n-1}, y_{n}, \phi\left(c^{r} t\right)\right)}-1 \\
\leq & (\alpha-\psi)^{n-r+1}\left(\frac{1}{M\left(f x_{r+1}, f x_{r+2}, \phi\left(\frac{c^{r} t}{c^{n-r+1}}\right)\right)}-1\right)
\end{aligned}
$$

making use of $y_{n}=f x_{n+1}(3.8)$ reduces to

$$
\begin{aligned}
& \frac{1}{M\left(y_{n-1}, y_{n}, \phi\left(c^{r} t\right)\right)}-1 \\
\leq & (\alpha-\psi)^{n-r+1}\left(\frac{1}{M\left(y_{r}, y_{r+1}, \phi\left(\frac{c^{r} t}{c^{n-r+1}}\right)\right)}-1\right) .
\end{aligned}
$$

Since $(\alpha-\psi)^{n}\left(a_{n}\right) \rightarrow 0$, whenever $a_{n} \rightarrow 0$ as $n \rightarrow \infty$, therefore for all $r>0$

$$
M\left(y_{n-1}, y_{n}, \phi\left(c^{r} t\right)\right) \rightarrow 1 \text { as } n \rightarrow \infty .
$$

Let $\epsilon>0$ be given, then by using properties of $\phi$ we can find $r>0$ such that $\phi\left(c^{r} t\right)<\epsilon$. It follows from (3.10) that $M\left(y_{n-1}, y_{n}, \epsilon\right) \rightarrow 1$ as $n \rightarrow \infty$, or

$$
M\left(y_{n}, y_{n+1}, \epsilon\right) \rightarrow 1 \text { as } n \rightarrow \infty
$$

By using triangular inequality, we have

$$
\begin{aligned}
& M\left(y_{n}, y_{n+p}, \epsilon\right) \\
\geq & M\left(y_{n}, y_{n+1}, \frac{\epsilon}{p}\right) * M\left(y_{n+1}, y_{n+2}, \frac{\epsilon}{p}\right) * \cdots * M\left(y_{n+p}, y_{n+p+1}, \frac{\epsilon}{p}\right)(p \text {-times }) .
\end{aligned}
$$

Taking $n \rightarrow \infty$ in above triangular inequality and using (3.11) for any integer $p$, we have $M\left(y_{n}, y_{n+p}, \epsilon\right) \rightarrow 1$, which implies that $\left\{y_{n}\right\}$ is a $G$-Cauchy sequence. As $(X, M, *)$ is $G$-complete, the sequence $\left\{y_{n}\right\}$ is convergent and hence there exists $z \in X$ such that $y_{n} \rightarrow z$ as $n \rightarrow \infty$, i.e., $y_{n}=T x_{n}=f x_{n+1} \rightarrow z$.

Let $v \in X$ such that $f v=z$, now we will show $v$ is a coincidence point of $f$ and $T$. Towards this, it is enough to show $T v=z$. Using triangular inequality from Definition 2.4,

$$
M(T v, z, \epsilon) \geq M\left(T v, y_{n}, \frac{\epsilon}{2}\right) * M\left(y_{n}, z, \frac{\epsilon}{2}\right) .
$$


With the help of Definition 2.11, we can find a $t_{2}>0$, such that $\phi\left(t_{2}\right)<\frac{\epsilon}{2}$. Since $y_{n} \rightarrow z$ as $n \rightarrow \infty$, then there exists $m \in N$ for all $n>m$, such that $M\left(y_{n}, z, \phi\left(t_{2}\right)\right)>0$. Therefore by the contraction (3.2) we have for $n>m$,

$$
\begin{aligned}
& \frac{1}{M\left(T v, y_{n}, \frac{\epsilon}{2}\right)}-1 \\
= & \frac{1}{M\left(T v, T x_{n}, \phi\left(t_{2}\right)\right)}-1 \\
\leq & \alpha\left(\frac{1}{M\left(f v, f x_{n+1}, \phi\left(\frac{t_{2}}{c}\right)\right)}-1\right)-\psi\left(\frac{1}{M\left(f v, f x_{n+1}, \phi\left(\frac{t_{2}}{c}\right)\right)}-1\right)
\end{aligned}
$$

on using (3.3) we obtain

$$
\begin{aligned}
& \frac{1}{M\left(T v, T x_{n+1}, \phi\left(t_{2}\right)\right)}-1 \\
\leq & \alpha\left(\frac{1}{M\left(f v, f x_{n+1}, \phi\left(\frac{t_{2}}{c}\right)\right)}-1\right)-\psi\left(\frac{1}{M\left(f v, f x_{n+1}, \phi\left(\frac{t_{2}}{c}\right)\right)}-1\right) .
\end{aligned}
$$

Proceeding limit as $n \rightarrow \infty$, utilizing $\phi(0)=0$ and continuity of $\psi, \alpha$ ones obtained,

$$
M\left(T v, y_{n}, \frac{\epsilon}{2}\right) \rightarrow 1 \text { as } n \rightarrow \infty .
$$

Taking $n \rightarrow \infty$ in (3.12), using (3.13) with continuity of $\psi, \alpha$, and the fact that $y_{n} \rightarrow z$ as $\mathrm{n} \rightarrow \infty$ we get, $M(T v, z, \epsilon)=1$ for every $\epsilon>0$. It follows that $T v=z$.

Next, we will prove that $z$ is a common fixed point of $f$ and $T$.

Notice that, the pair $(f, T)$ are weakly compatible therefore $f z=f T v=$ $T f v=T z$. Now our aim is to show $T z=z$.

Again

$$
M(T z, z, \epsilon) \geq M\left(T z, y_{n}, \frac{\epsilon}{2}\right) * M\left(y_{n}, z, \frac{\epsilon}{2}\right) .
$$

On using the property of $\phi$-function, we can find a $t_{3}>0$, such that $\phi\left(t_{3}\right)<$ $\frac{\epsilon}{2}$. Also $y_{n} \rightarrow z$ as $n \rightarrow \infty$, hence there exists $m \in N$ such that for all $n>m$, $M\left(y_{n}, z, \phi\left(t_{3}\right)\right)>0$. Then for $n>m$,

$$
\begin{aligned}
& \frac{1}{M\left(T z, y_{n}, \frac{\epsilon}{2}\right)}-1 \\
= & \frac{1}{M\left(T z, T x_{n}, \phi\left(t_{3}\right)\right)}-1 \\
\leq & \alpha\left(\frac{1}{M\left(f z, f x_{n+1}, \phi\left(\frac{t_{3}}{c}\right)\right)}-1\right)-\psi\left(\frac{1}{M\left(f z, f x_{n+1}, \phi\left(\frac{t_{3}}{c}\right)\right)}-1\right)
\end{aligned}
$$

is obvious from (3.2), now applying (3.3) we get

$$
\frac{1}{M\left(T z, T x_{n+1}, \phi\left(t_{3}\right)\right)}-1
$$




$$
\leq \alpha\left(\frac{1}{M\left(f z, f x_{n+1}, \phi\left(\frac{t_{3}}{c}\right)\right)}-1\right)-\psi\left(\frac{1}{M\left(f z, f x_{n+1}, \phi\left(\frac{t_{3}}{c}\right)\right)}-1\right) .
$$

Proceeding the limit $n \rightarrow \infty$, utilizing $\phi(0)=0$ and continuity of $\psi, \alpha$, we obtain

$$
M\left(T z, y_{n}, \frac{\epsilon}{2}\right) \rightarrow 1 \text { as } n \rightarrow \infty
$$

In the similar manner, making $n \rightarrow \infty$ in (3.14), using (3.15) with continuity of $\psi, \alpha$ and the fact that $y_{n} \rightarrow z$ as $n \rightarrow \infty$ it is clear that, $M(T z, z, \epsilon)=1$ for every $\epsilon>0$, which gives $T z=z$. Thus, we have shown that $f z=T z=z$ which implies that $z$ is a common fixed point of $f$ and $T$.

Finally, it remains to prove the uniqueness of $z$. Let, if possible $z, z^{\prime}$ be two fixed points of $f$ and $T$, by the properties of $\phi$ there exists $s>0$ such that $M\left(z, z,^{\prime} \phi(s)\right)>0$, then by applying (3.2) we obtain the following equation

$$
\begin{aligned}
& \frac{1}{M\left(z, z,^{\prime} \phi(c s)\right)}-1 \\
= & \frac{1}{M\left(T z, T z,^{\prime} \phi(c s)\right)}-1 \\
\leq & \alpha\left(\frac{1}{M\left(f z, f z,^{\prime} \phi(s)\right)}-1\right)-\psi\left(\frac{1}{M\left(f z, f z,^{\prime} \phi(s)\right)}-1\right) \\
\leq & \alpha\left(\frac{1}{M\left(f z, f z,^{\prime} \phi(s)\right)}-1\right)-\psi\left(\frac{1}{M\left(f z, f z,^{\prime} \phi(s)\right)}-1\right) .
\end{aligned}
$$

Also $M\left(f z, f z,^{\prime} \phi(s)\right)>0$ implies $M\left(f z, f z,^{\prime} \phi\left(\frac{s}{c}\right)\right)>0$ here on replacing $s$ by $\frac{s}{c}$ in above equation $(3.16)$

$$
\frac{1}{M\left(z, z,^{\prime} \phi(s)\right)}-1 \leq \alpha\left(\frac{1}{M\left(f z, f z,^{\prime} \phi\left(\frac{s}{c}\right)\right)}-1\right)-\psi\left(\frac{1}{M\left(f z, f z,^{\prime} \phi\left(\frac{s}{c}\right)\right)}-1\right) .
$$

Following the above process $n$ times the equation becomes

$$
\frac{1}{M\left(z, z,^{\prime} \phi(s)\right)}-1 \leq(\alpha-\psi)^{n}\left(\frac{1}{M\left(f z, f z,^{\prime} \phi\left(\frac{s}{c}\right)\right)}-1\right) .
$$

It is easy to see that $(\alpha-\psi)^{n}\left(a_{n}\right) \rightarrow 0$ as $n \rightarrow \infty$, which implies that

$$
M\left(z, z^{\prime}, \phi(s)\right)=1 \quad \forall s>0 .
$$

Again from (3.16), it follows that $M\left(z, z,^{\prime} \phi(c s)\right)>0$. The same argument will hold when $s$ is replaced by $c s$ which gives $M\left(z, z,{ }^{\prime} \phi(c s)\right)=1$. In fact, in general $\left.M\left(z, z,^{\prime} \phi\left(c^{n} s\right)\right)\right)=1$ for all $n \in N \cup\{0\}$. Clearly, from the properties of $\phi$ for any given $\epsilon>0$ there exists $r \in N \cup\{0\}$ such that $\phi\left(c^{r} s\right)<\epsilon$, therefore from the above analysis we get $M(z, z, \epsilon)=1$ for all $\epsilon>0$, which implies $z=z^{\prime}$. This establishes the uniqueness of fixed point. 
Example 3.1. Let $X=M(x, y, *)$ be a complete fuzzy metric space, where $X=\left\{x_{1}, x_{2}, x_{3}\right\}, a * b=\min \{a, b\}$ and $M(x, y, t)$ is defined as

$$
\begin{gathered}
M\left(x_{2}, x_{3}, t\right)=M\left(x_{3}, x_{2}, t\right)=\left\{\begin{array}{cl}
0, & \text { if } t=0 \\
0.8, & \text { if } 0<t<3 \\
1, & \text { if } t \geq 3
\end{array}\right\}, \\
M\left(x_{1}, x_{3}, t\right)=M\left(x_{3}, x_{1}, t\right)=M\left(x_{1}, x_{2}, t\right)=M\left(x_{2}, x_{1}, t\right)=\left\{\begin{array}{ll}
0, & \text { if } t=0 \\
1, & \text { if } t>0
\end{array}\right\},
\end{gathered}
$$

where $T, f: X \rightarrow X$ are defined as $T\left(x_{1}\right)=x_{1} ; T\left(x_{2}\right)=x_{3} ; T\left(x_{3}\right)=x_{1}$ and $f\left(x_{1}\right)=x_{1} ; f\left(x_{2}\right)=x_{3} ; f\left(x_{3}\right)=x_{2}$. If we take $\phi(t)=t, \psi(t)=\frac{t}{6}$, and $\alpha(t)=t$ and $c=\frac{1}{2}$.

Clearly, together with this $f, T$ satisfies all the conditions of Theorem 3.1 and $(f, T)$ are also weakly compatible. Hence $f, T$ have a unique common fixed point at $x_{1}$.

Acknowledgement. Author Amit Kumar is heartily acknowledged to Ms. Pranjali for providing her valuable suggestion to write this manuscript.

\section{References}

[1] C. T. Aage and B. S. Choudhury, Some fixed point results in fuzzy metric spaces using a control function, to appear.

[2] C. T. Aage and J. N. Salunke, On fixed point theorems in fuzzy metric spaces using a control function, Int. J. Nonlinear Anal. Appl. 2 (2011), no. 1, 50-57.

[3] B. S. Choudhury and K. Das, A new contraction principle in Menger spaces, Acta Math. Sin. (Engl. Ser.) 24 (2008), no. 8, 1379-1386.

[4] B. S. Choudhury, P. N. Dutta, and K. Das, A fixed points result in Menger space using a real function, Acta Math. Hungar. 122 (2009), no. 3, 203-216.

[5] P. N. Dutta and B. S. Choudhury, A generalisation of contraction principle in metric spaces, Fixed Point Theory and Applications 2008 (2008), Article ID 406368, doi: $10.1155 / 2008 / 406368$.

[6] P. N. Dutta, B. S. Choudhury, and Krishnapada Das, Some fixed point results in Menger spaces using a control function, Surv. Math. Appl. 4 (2009), 41-52.

[7] A. George and P. Veeramani, On some results of analysis for fuzzy metric spaces, Fuzzy Sets and Systems 90 (1997), no. 3, 365-368.

[8] - On some results in fuzzy metric spaces, Fuzzy Sets and Systems 64 (1994), no. 3, 395-399.

[9] V. Gregori and S. Romaguerab, Some properties of fuzzy metric spaces, Fuzzy Sets and Systems 115 (2000), no. 3, 485-489.

[10] V. Gregori and A. Sapena, On fixed point theorems in fuzzy metric spaces, Fuzzy Sets and Systems 125 (2002), no. 2, 245-252.

[11] M. S. Khan, M. Swaleh, and S. Sessa, Fixed point theorems by altering distances between the points, Bull. Austral. Math. Soc. 30 (1984), no. 1, 1-9.

[12] I. Kramosil and J. Michalek, Fuzzy metric and statistical metric spaces, Kybernetika (Prague) 11 (1975), no. 5, 326-334.

[13] D. Mihet, Multivalued generalizations of probabilistic contractions, J. Math. Anal. Appl. 304 (2005), no. 2, 464-472.

[14] - On fuzzy contractive mapping in fuzzy metric, Fuzzy Sets and Systems 158 (2007), no. 8, 915-921.

[15] B. Schweizer and A. Sklar, Statistical metric spaces, Pacific J. Math. 10 (1960), 314-334. 
[16] R. Vasuki and P. Veeramani, Fixed point theorems and Cauchy sequences in fuzzy metric spaces, Fuzzy Sets and Systems 135 (2003), no. 3, 415-417.

Amit Kumar

Department of Mathematics

National Institute of Technology

HAMIRPUR - 177005, INDIA

E-mail address: amitsu48@gmail.com

Ramesh Kumar Vats

Department of Mathematics

National Institute of Technology

HAMIRPUR - 177005, INDIA

E-mail address: ramesh_vats@rediffmail.com 